\title{
A minimum adjustment cost feedback mechanism based consensus model for group decision making under social network with distributed linguistic trust
}

\author{
Jian Wu ${ }^{\mathrm{a}}$, Lifang Dai ${ }^{\mathrm{b}}$, Francisco Chiclana $^{\mathrm{c}}$, Hamido Fujita ${ }^{\mathrm{d}}$, Enrique Herrera-Viedma ${ }^{\mathrm{e}} \mathrm{f}$ \\ ${ }^{a}$ School of Economics and Management, Shanghai Maritime University, Shanghai201306, China \\ ${ }^{b}$ School of Economics and Management, Zhejiang Normal University, Jinhua, China \\ ${ }^{c}$ Centre for Computational Intelligence, Faculty of Technology, De Montfort University, Leicester, UK \\ ${ }^{d}$ Iwate Prefectural University, Takizawa, Iwate, Japan \\ ${ }^{e}$ Department of Computer Science and Artificial Intelligence, University of Granada, Granada, Spain \\ ${ }^{f}$ Department of Electrical and Computer Engineering, Faculty of Engineering, King Abdulaziz University, Jeddah 21589, \\ Saudi Arabia
}

\begin{abstract}
A theoretical feedback mechanism framework to model consensus in social network group decision making (SN-GDM) is proposed with following two main components: (1) the modelling of trust relationship with linguistic information; and (2) the minimum adjustment cost feedback mechanism. To do so, a distributed linguistic trust decision making space is defined, which includes the novel concepts of distributed linguistic trust functions, expectation degree, uncertainty degrees and ranking method. Then, a social network analysis (SNA) methodology is developed to represent and model trust relationship between a networked group, and the trust in-degree centrality indexes are calculated to assign an importance degree to the associated user. To identify the inconsistent users, three levels of consensus degree with distributed linguistic trust functions are calculated. Then, a novel feedback mechanism is activated to generate recommendation advices for the inconsistent users to increase the group consensus degree. Its novelty is that it produces the boundary feedback parameter based on the minimum adjustment cost optimisation model. Therefore, the inconsistent users are able to reach the threshold value of group consensus incurring a minimum modification of their opinions or adjustment cost, which provides the optimum balance between group consensus and individual independence. Finally, after consensus has been achieved, a ranking order relation for distributed linguistic trust functions is constructed to select the most appropriate alternative of consensus.
\end{abstract}

Keywords: Group Decision Making, Feedback Mechanism, Minimum Adjustment Optimization Model, Consensus, Social Network Analysis, Distributed Linguistic Trust.

\section{Introduction}

This paper focuses on group decision making (GDM) where users construct preference relations by comparing a finite set of alternatives $X=\left\{x_{1}, x_{2}, \ldots, x_{n}\right\}$, and by aggregating them into a collective one and applying a selection process a common solution is derived $[1,2,34]$. An issue that needs to

Email addresses: jyajian@163.com (Jian Wu), dlf20110913@163.com (Lifang Dai), chiclana@dmu.ac.uk (Francisco Chiclana), HFujita-799@acm.org (Hamido Fujita), viedma@decsai.ugr.es (Enrique Herrera-Viedma) 
be addressed in GDM is the inconsistency of information provided by the users, which normally arises from the different background and knowledge each individual user has on the decision making problem under consideration $[6,12,20,31]$. Obviously, it is preferable that the group of users achieves consensus (agreement) before applying an appropriate aggregation procedure to derive the final solution to the decision problem. This topic has attracted the interest of many researchers in the field of GDM, and group interaction consensus model has been proved to be an effective approach to reduce or eliminate inconsistency $[23,32,38,46]$. This model usually involves a feedback mechanism to advice group inconsistent users, i.e. users with a consensus level below a group consensus threshold value, on how to modify their preference values $[5,21,42]$. In any case, the effectiveness of a feedback mechanism is determined by the quality of its recommendation advices, which will ultimately affect their acceptance by the inconsistent users.

As stated above, one key issue to be addressed in consensus within GDM is how to design and develop an effective feedback mechanism. Most of the different feedback mechanisms proposed in this field $[5,21,42]$ produce recommendation advices by implementing a linear weighted approach with a fixed or static feedback parameter. As pointed out by Wu and Chiclana in [41], these feedback mechanisms share the common limitation of forcing the inconsistent users to implement the recommendation advices without considering whether they like them or not. We believe that the main issues associated with this approach are that the feedback parameter is discretionary selected, and that the inconsistent users have no idea of their resultant new consensus status and cost associated with modifying their opinions, which it will be referred to as adjustment cost herein, when implementing the feedback advices. A more reasonable policy is possible and desirable by which the inconsistent users can decide to implement or not the recommendation advices provided to him/her based on the affordability of their adjustment cost $[17,18,44,47]$. Usually, the higher the feedback parameter value is in the aforementioned linear weighted approach, the higher the adjustment cost the inconsistent users will bear. On the other hand, the inconsistent users will not reach the threshold value of group consensus if the feedback parameter is not high enough. Hence, how to select the optimal feedback parameter to balance the individual adjustment cost and the group consensus reaching is a challenge to address in the GDM interaction consensus process. To our knowledge, there are few researchers paying attention to the research of this issue so far. Therefore, the first object of this article is to investigate a minimum adjustment cost feedback mechanism in GDM. To do that, an optimal model is proposed to determine the boundary feedback parameter to make the inconsistent users arrive at a balance between group consensus and individual adjustment cost, which can be used as a rational argument for the derived feedback recommendation advices to be accepted and, ultimately, implemented by the inconsistent users to achieve the threshold value of group consensus with minimum adjustment cost.

Another issue to be addressed in GDM is the heterogeneous problem between group users $[4,33]$. This is usually resolved by assigning importance degrees to users beforehand based on the assumption 
that these stem from some reliable sources of information $[13,16,50,54]$. However, in most cases, this assumption may be unrealistic or improbable because there might not exist historical records of interaction between the users in the group. Considering that a new trend of GDM today is related to social network and that individuals make decisions relying on the opinions from their network of friends and/or acquaintances under social network [7, 25, 27, 35, 36], a group decision making model within a social network framework would be of importance. Community review websites where users comment on consumer products and give a rating to the products and the reviews written by others can be modelled as a social network. Using this interaction information, a trust relationship between users can be constructed, which should be considered as a reliable resource in the interaction process of GDM [22]. Therefore, group decision making under social network can have the historical interaction relationship between members of a group, which provides a completely different approach to the heterogeneity of users when compared to the classical GDM model mentioned above. Thus, within the social network group decision making (SN-GDM ) framework it could be interesting to provide new reliable sources to acquire users weight information base on trust relationship.

Traditionally, trust is usually expressed as a binary or crisp relation as shown in Table 1, i.e. 'trusting' and 'not trusting'. However, in many situations, it may not be suitable to represent the relation in a crisp way because it is not clear cut defined. Notice that in real life too, trust is often interpreted as a gradual concept with people trusting someone 'high', 'middle' and/or 'low' [8]. Considering that in social network, more often than not, users express their preferences using natural language, it could be more realistic and practice in GDM to assume that linguistic information is used by users to express their trust preferences [26, 28, 30, 39, 53]. Thus, in this paper, we assume the use of the distributed linguistic trust to establish the trust relationship between group users. Both the operational laws and weighted averaging operator of distributed linguistic trust are explored in detail. Afterwards, the expectation degree and uncertainty degrees are given, and a ranking method of distributed linguistic trust functions is investigated. Finally, the importance degree of each user is determined by trust relationship between the group in social network.

The rest of this paper is set out as follows: Section 2 provides the preliminaries needed for the rest of the paper regarding social network group decision making (Section 2.1) and linguistic distribution (Section 2.2). Section 3 firstly introduces the concept of distributed linguistic trust functions (Section 3.1) and its associated operational laws, weighted averaging operator, expectation and uncertainty degrees and order relation. Afterwards, the Social Network with distributed linguistic trust is constructed (Section 3.2). Thus, trust in-degree centrality indexes of each user are calculated to assign the importance degrees to the associated users. Section 4 proposes the concept of consensus degree on three levels of opinion expressed by distributed linguistic trust information, and the inconsistent users and elements values that contribute less to consensus are identified. Then, a minimum adjustment cost feedback mechanism is activated to produce recommendation advices to the inconsistent users so 
Table 1: Different representation schemes in Social Network Analysis

$$
A=\left(\begin{array}{cccccc}
0 & 1 & 1 & 1 & 1 & 0 \\
0 & 0 & 0 & 0 & 1 & 0 \\
0 & 1 & 0 & 0 & 0 & 0 \\
0 & 0 & 1 & 0 & 1 & 1 \\
0 & 0 & 1 & 0 & 0 & 1 \\
0 & 0 & 1 & 0 & 0 & 0
\end{array}\right)
$$

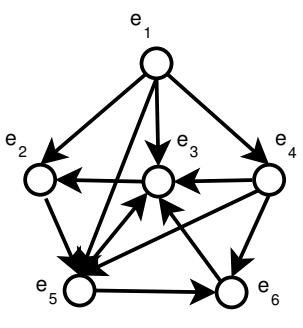

that higher consensus is achieved. In detail, it establishes an optimal model to provide the boundary feedback parameter for balancing consensus and adjustment cost. Finally, in Section 5, an analysis between the proposed consensus model and traditional one is given, and then conclusions are drawn.

\section{Preliminaries}

\subsection{Social Network Group Decision Making}

Nowadays, group decision making are usually carried out within a social network framework in which individuals rely on the opinions and social appraisal support from their close friends or people with similar interests. A popular example is the consumer review site Epinions.com, where users can write reviews about consumer products and assign a rating to the products and the reviews. In essence, Epinions.com is a social network, i.e. an association of people drawn together by family, work or hobby. Then the social relationship should be taken into account in the interaction process of GDM [36].

Social network analysis (SNA) is a useful tool to study the relationships between social entities like families, corporations or nations [40], which help us to examine the structural and locational properties including centrality, prestige, structural balance and trust relationship, among others (in Table 1). Consequently, a SNA based methodology could be used to model the concept of trust degree (TD) as well as to make possible its measurement to reflect the actual trust relationships between users in a group [11, 43, 45, 48]. Therefore, this social network group decision making (SN-GDM) is a complete new type of decision making problem because it takes into account the trust relationship in the process of reaching consensus while the traditional GDM models neglect it. Several methods are developed to enrich the field of SN-GDM such as: social network based aggregation model [43], trust induced recommendation mechanism [29], trust propagation models [48], leadership based consensus [10], opinion based group recommender [4], etc. The aim of this article is to investigate a minimum adjustment cost feedback mechanism based consensus for SN-GDM. 


\subsection{Linguistic Distribution}

The linguistic approach has been regarded a useful technique to represent qualitative aspects by linguistic variables. For instance, $\mathrm{Xu}$ [51] proposed a continuous linguistic model as: Let $S=$ $\left\{s_{\alpha} \mid \alpha=-t, \ldots .-1,0,1 \ldots, t\right\}$ be a definite and totally ordered discrete term set in which $S$ represents a possible value for a linguistic variable. For example, $S$ with nine terms can be defined as:

$$
S=\left\{\begin{array}{c}
s_{-4}=\text { extremely poor } ; s_{-3}=\text { very poor } ; s_{-2}=\text { poor } \\
s_{-1}=\text { slightly poor } ; s_{0}=\text { fair } ; s_{1}=\text { slightlygood } \\
s_{2}=\text { good } ; s_{3}=\text { very good } ; s_{4}=\text { extremely good }
\end{array}\right\}
$$

For any two linguistic terms $s_{\alpha}, s_{\beta} \in S, s_{t}=\operatorname{Max}\left\{s_{\alpha}\right\}$ and $\mu, \mu_{1}, \mu_{2}>0$, they have the following operational laws [51]:

(1) $s_{\alpha} \oplus s_{\beta}=s_{\alpha+\beta}=\operatorname{Min}\left\{s_{\alpha+\beta}, s_{t}\right\}$;

(2) $s_{\alpha} \oplus s_{\beta}=s_{\beta} \oplus s_{\alpha}$;

(3) $\mu s_{\alpha}=s_{\mu \alpha}$;

(4) $\left(\mu_{1}+\mu_{2}\right) s_{\alpha}=\mu_{1} s_{\alpha} \oplus \mu_{2} s_{\alpha}$;

(5) $\mu\left(s_{\alpha} \oplus s_{\beta}\right)=\mu s_{\alpha} \oplus \mu s_{\beta}$;

Recently, Zhang et al.[53] proposed the concept of the distribution assessment in a linguistic term set in which symbolic proportions are assigned to all the linguistic terms.

Definition 1. Let $S=\left\{s_{-t}, \ldots, s_{t}\right\}$ be a linguistic term set. Let $m=\left\{\left(s_{k}, \beta_{k}\right) \mid k=-T, \ldots T\right\}$, where $s_{k} \in S, \beta_{k} \geq 0, \sum_{k=-T}^{T} \beta_{k}=1$ and $\beta_{k}$ is the symbolic proportion of $s_{k}$. Then $m$ is called a distribution assessment of $S$.

To achieve an ordering of distribution assessment of a linguistic term set, Zhang et al.[53] introduced the definition of expectation function as:

Definition 2. Let $m=\left\{\left(s_{k}, \beta_{k}\right) \mid k=-T, \ldots T\right\}$, where $s_{k} \in S, \beta_{k} \geq 0, \sum_{k=-T}^{T} \beta_{k}=1$ be a distribution assessment of $S$. The expectation of $m$ is defined as follows:

$$
E(m)=\sum_{k=-T}^{T} \beta_{k} s_{k}
$$

Obviously, the distribution assessment of a linguistic term set has advantage to deal with uncertainty information because of the symbolic proportions. This idea also can be used to investigate the distribution linguistic trust functions (DLTFs) in the following section. 


\section{Social Network with Distributed Linguistic Trust}

\subsection{Definition of Distributed Linguistic Trust}

The main three elements in SNA analysis are: the set of actors, the relations themselves, and the actor attributes (see Table 1). The important network concepts in a unify manner using the three different and possible representation schemes are:

- Sociometric: relational data are often presented in two-ways matrices called sociomatrix.

- Graph theoretic: the network is viewed as a graph consisting of nodes joined by lines.

- Algebraic: allows to distinguish several distinct relations and represent combinations of relations.

However, the above sociomatrix is a binary or crisp relation, and then it may not be suitable to model uncertainty in trust relationship representation in social network, which is indeed often interpreted as a probabilistic phenomenon: humans do not merely express the terms of 'trusting' and 'not trusting', but rather trust someone 'high'or 'middle' and 'low'. [4, 10]

The above continuous linguistic model can be used to describe the probabilistic phenomenon in trust relationship under social network. To do so, this article defines the concept of distribution linguistic trust.

Definition 3. Let $H=\left\{H_{\alpha} \mid \alpha=1 \ldots, \xi\right\}$ be a linguistic term set. Then, the distributed linguistic trust functions DLTFs is defined as:

$$
T=\left\{\left(H_{\alpha}, \varphi_{\alpha}\right) \mid \alpha=1, \ldots, \xi\right\}
$$

where $H_{\alpha} \in H$ is a linguistic term, and $\varphi_{\alpha}$ is the symbolic proportion of $H_{\alpha} \cdot \sum_{\alpha=1}^{\xi} \varphi_{\alpha}=1$, and $\varphi_{\alpha} \geq 0$.

For any two distributed linguistic trust functions $T^{1}, T^{2} \in T$ and $\mu, \mu_{1}, \mu_{2}>0$, their operational laws are as follows:

(1) $T^{1} \oplus T^{2}=\left\{\left(H_{\alpha},\left(\varphi_{\alpha}^{1}+\varphi_{\alpha}^{2}\right)\right) \mid \alpha=1, \ldots, \xi\right\}$;

(2) $T^{1} \oplus T^{2}=T^{2} \oplus T^{1}$;

(3) $\mu T=\left\{\left(H_{\alpha}, \mu \varphi_{\alpha}\right) \mid \alpha=1, \ldots, \xi\right\}$;

(4) $\left(\mu_{1}+\mu_{2}\right) T=\mu_{1} T \oplus \mu_{2} T$;

(5) $\mu\left(T^{1} \oplus T^{2}\right)=\mu T^{1} \oplus \mu T^{2}$;

According to the above operational laws, we can develop the following weighted averaging operator for aggregating distributed trust functions in group decision making. 
Definition 4. Let $\left\{T^{1}, \ldots, T^{n}\right\}$ be a set of distributed linguistic trust functions and $\omega=\left(\omega_{1}, \omega_{2}, \ldots ., \omega_{n}\right)$ be an associated weighting vector, $\omega_{j}>0$ and $\sum_{j=1}^{n} \omega_{j}=1$, where $T^{j}=\left\{\left(H_{\alpha}, \varphi_{\alpha}^{j}\right) \mid \alpha=1, \ldots, \xi\right\}$, $j=1,2, \ldots, n$. Then, the weighted averaging operator of distributed trust function can be computed as

$$
D T W A_{\omega}\left(T^{1}, \ldots, T^{\mathrm{n}}\right)=\left\{\left(H_{\alpha}, \bar{\varphi}_{\alpha}\right) \mid \alpha=1, \ldots, \xi\right\}
$$

where $\bar{\varphi}_{\alpha}=\sum_{j=1}^{n} \omega_{j} \varphi_{\alpha}^{j}$.

To make a ranking order of distributed linguistic trust functions, the expectation degree and uncertainty degree are defined as follows, respectively.

Definition 5. Let $T=\left\{\left(H_{\alpha}, \varphi_{\alpha}\right) \mid \alpha=1, \ldots, \xi\right\}$ be a distributed linguistic trust function, where $H_{\alpha} \in H, \sum_{\alpha=1}^{\xi} \varphi_{\alpha}=1$, and $\varphi_{\alpha} \geq 0$, be a distributed assessment of $H$. The expectation degree of $T$ can be defined as this:

$$
E(T)=\sum_{\alpha=1}^{\xi} H_{\alpha} \times \varphi_{\alpha}=H_{\sum_{\alpha=1}^{\xi} \alpha \varphi_{\alpha}}
$$

For brevity, we denote $\sum$ as the $\oplus$ operation of multiple linguistic terms in this paper. The larger the value of $E(T)$, the more the degree of score of $T$.

Definition 6. Let $T=\left\{\left(H_{\alpha}, \varphi_{\alpha}\right) \mid \alpha=1, \ldots, \xi\right\}$ be a distributed linguistic trust function, where $H_{\alpha} \in H, \sum_{\alpha=1}^{\xi} \varphi_{\alpha}=1$, and $\varphi_{\alpha} \geq 0$, be a distributed assessment of $H$. The uncertainty degree of $T$ can be defined as this:

$$
U(T)=\frac{1}{\xi} \sum_{\alpha=1}^{\xi}\left(\alpha \varphi_{\alpha}-\sum_{\alpha=1}^{\xi} \alpha \varphi_{\alpha}\right)^{2}
$$

The bigger the value of $U(T)$, the smaller the degree of score of $T$.

In a group decision making problem under social network environment, distributed linguistic trust are needed to describe the relationship between group users, and then to distinguish the highest trusted decision maker within their social network. Therefore, an approach for ranking linguistic trust functions should be proposed. To do that, this article first introduces the definition of distributed linguistic trust decision space as follows:

Definition 7. (Distributed Linguistic Trust Decision Space(TDS). A distributed linguistic trust decision space

$$
T D S^{\square}=\left(\Lambda, \leq_{E(T)}, \leq_{U(T)}\right)
$$

consists of the set of distributed linguistic trust functions $\Lambda$, a expectation ordering $\leq_{E(T)}$ and a uncertainty ordering $\leq_{U(T)}$ that have the following properties

$$
\begin{aligned}
& T^{1} \leq_{E(T)} T^{2} \text { iff } E\left(T^{1}\right) \leq E\left(T^{2}\right) \\
& T^{1} \leq_{U(T)} T^{2} \text { iff } U\left(T^{1}\right) \geq U\left(T^{2}\right)
\end{aligned}
$$


In a $T D S, E(T)$ is used to calculate the degree of strict trust about DLTFs while $U(T)$ is for determining the uncertainty contained in the corresponding DLTFs. Then, their role for ranking DLTFs is similar to the mean and the variance in Statistics. In detail, a order relation on the set of DLTFs is defined by $\Lambda$ :

Definition 8 (Order Relation of DLTFs). Given two DLTFs, $T^{1}$ and $T^{2}$, it can say that $T^{1}$ precedes $T^{2}$

$$
T^{1} \prec T^{2}
$$

if and only if one of the following conditions is true:

1. $E\left(T^{1}\right)<E\left(T^{2}\right)$

2. $E\left(T^{1}\right)=E\left(T^{2}\right) \wedge U\left(T^{1}\right)>U\left(T^{2}\right)$

The next result guarantees that the order relation $\prec$ is a strict order.

Theorem 1 (Strict Order of DLTFs). The relation $\prec$ on the set of DLTFs $\Lambda$ is:

1. Irreflexive: $\forall T: T \prec T$ does not hold.

2. Asymmetric: $\forall T^{1}, T^{2}$ : if $T^{1} \prec T^{2}$, then $T^{2} \prec T^{1}$ does not hold.

3. Transitive: $\forall T^{1}, T^{2}, T^{3}$ : if $T^{1} \prec T^{2}$ and $T^{2} \prec T^{3}$, then $T^{1} \prec T^{3}$.

Proof. Items 1. and 2. are obvious from Definition 8. Then, we just need prove the transitivity property. Starting with $T^{1} \prec T^{2}$, from Definition 8, we get two possible cases:

1. $E\left(T^{1}\right)<E\left(T^{2}\right)$. In this case, it is clear that $E\left(T^{2}\right)<E\left(T^{3}\right)$, no matter which condition is true for $T^{1} \prec T^{3}$.

2. $E\left(T^{1}\right)=E\left(T^{2}\right) \wedge U\left(T^{1}\right)>U\left(T^{2}\right)$. Because $T^{2} \prec T^{3}$ then one of the following is true:

(a) $E\left(T^{2}\right)<E\left(T^{3}\right)$. In this case, it has that $E\left(T^{2}\right)<E\left(T^{3}\right)$ and then it is $T^{1} \prec T^{3}$.

(b) $E\left(T^{2}\right)=E\left(T^{3}\right) \wedge U\left(T^{2}\right)>U\left(T^{3}\right)$. Then, it can conclude that $E\left(T^{1}\right)=E\left(T^{3}\right) \wedge U\left(T^{1}\right)>$ $E\left(T^{3}\right)$ and therefore it is $T^{1} \prec T^{3}$.

We conclude that $T^{1} \prec T^{2}$ and $T^{2} \prec T^{3}$ implies $T^{1} \prec T^{3}$.

We have the following twofold usage of the order relation of DLTFs: (1) build the trust relationship from social network between group users and then use this trust relationship as a resource to assign weights to user; (2) distinguish the best alternative from the collective decision making matrices with DLTFs. 


\subsection{Building Trust Relationship by Social Network with DLTFs}

Definition 9. A distributed linguistic trust sociomatrix $S_{L}$ on $E$ is a relation in $E \times E$ with membership function $\mu_{S_{L}}: E \times E \rightarrow\left\{H_{\alpha}, \varphi_{\alpha}\right\}$,

$$
\mu_{S_{L}}\left(e_{s}, e_{k}\right)=T_{s k}
$$

where $E=\left\{e_{1}, \ldots, e_{m}\right\}$ be the set of nodes, $T_{s k}=\left\{\left(H_{\alpha}, \varphi_{\alpha}\right) \mid \alpha=1, \ldots, \xi\right\}$ is a distributed linguistic trust function, which means the trust degree from user $e_{s}$ to user $e_{k}$.

In a directed graph, the in-degree of centrality can be used to determine the importance of nodes in the network:

Definition 10. Let $G=(E, L, \omega)$ be a directed graph, $E=\left\{e_{1}, \ldots, e_{m}\right\}$ be the set of nodes and $L=\left\{l_{1}, \ldots, l_{q}\right\}$ be the set of directed lines, or arcs, between pairs of nodes and the set of distributed linguistic trust assessments $\omega=\left\{\omega_{1}^{L}, \ldots, \omega_{q}^{L}\right\}$ attached to the lines (or arcs), $S_{L}=\left(T_{s k}\right)_{m \times m}$ be the sociomatrix associated with the graph $G=(E, L, \omega)$, then the relative node in-degree centrality index obtained from the sociomatrix is computed as follows:

$$
C_{D}^{L}\left(e_{k}\right)=\frac{1}{m-1} \sum_{s=1}^{m} T_{s k}
$$

Obviously, the in-degree centrality index reflects the corresponding importance degree of users. Then, we can define the importance degree of each user in the group as follows:

Definition 11 (Importance Degree). Let $G=(E, L, \omega)$ be a directed graph representing the trust relationship between the group of users $E=\left\{e_{1}, \ldots, e_{m}\right\}$ and $\left\{C_{D}^{L}\left(e_{1}\right), \ldots, C_{D}^{L}\left(e_{h}\right)\right\}$ be the set of indegree centrality indexes. The importance degree of user $e_{h}$ can be expressed as:

$$
w_{k}=\frac{E\left(G_{D}^{L}\left(e_{k}\right)\right)}{\sum_{k=1}^{l} E\left(G_{D}^{L}\left(e_{k}\right)\right)}
$$

where $E\left(G_{D}^{L}\left(e_{k}\right)\right)$ is the expectation of distributed linguistic trust function as per expression (3).

Example 1. A group of five different users $\left\{e_{1}, e_{2}, e_{3}, e_{4}, e_{5}\right\}$ under a social network have the trust relationship in Figure 1. For simplicity, we set the linguistic scale as $H=\left\{h_{1}=l o w ; h_{2}=\right.$ middle; $\left.h_{3}=h i g h\right\}$, 
then the distributed linguistic trust sociomatrix $S_{L}$ are constructed as follows:

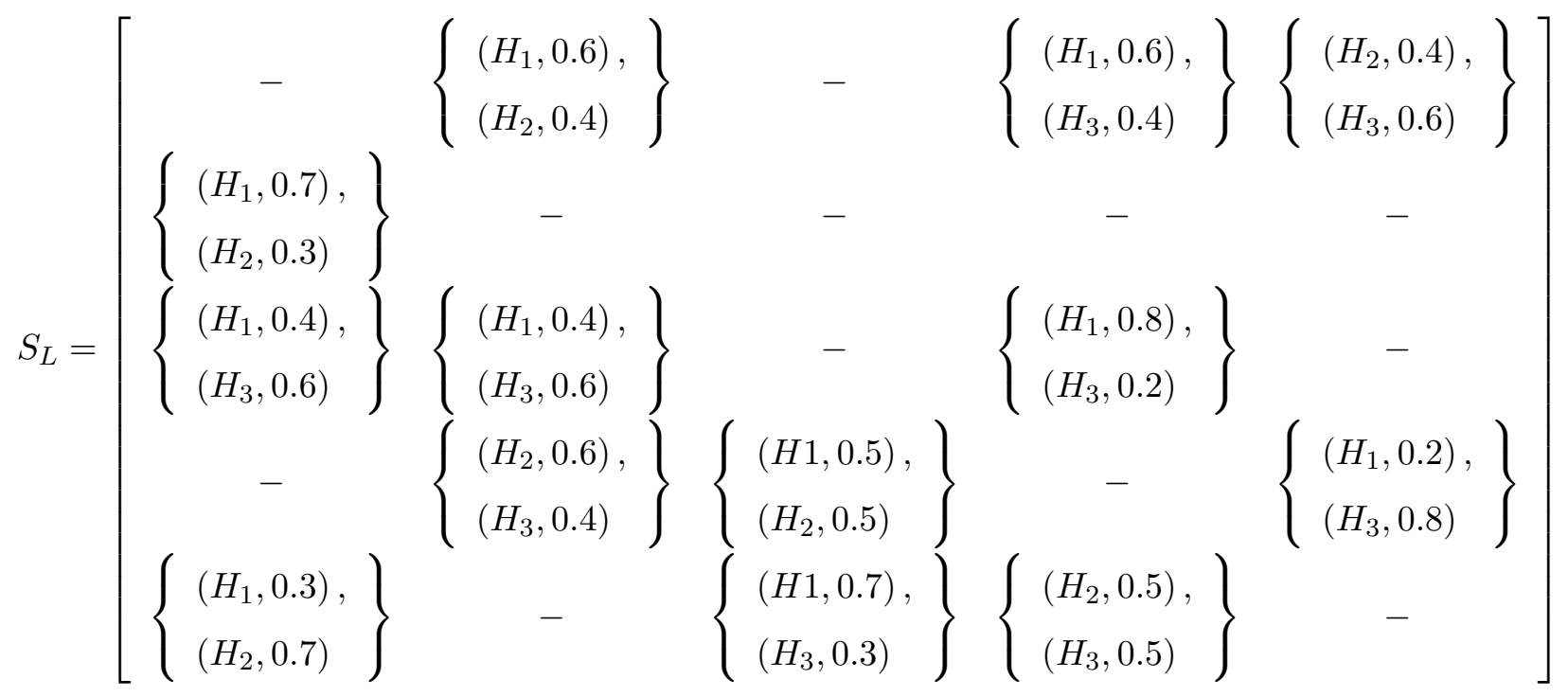

By expression (6), the following trust in-degree centrality indexes are calculated as:

$$
\begin{gathered}
C_{D}^{L}\left(e_{1}\right)=\left\{\left(H_{1}, 0.47\right),\left(H_{2}, 0.33\right),\left(H_{3}, 0.2\right)\right\} ; C_{D}^{L}\left(e_{2}\right)=\left\{\left(H_{1}, 0.33\right),\left(H_{2}, 0.33\right),\left(H_{3}, 0.34\right)\right\} \\
C_{D}^{L}\left(e_{3}\right)=\left\{\left(H_{1}, 0.60\right),\left(H_{2}, 0.25\right),\left(H_{3}, 0.15\right)\right\} ; C_{D}^{L}\left(e_{4}\right)=\left\{\left(H_{1}, 0.47\right),\left(H_{2}, 0.16\right),\left(H_{3}, 0.37\right)\right\} ; \\
C_{D}^{L}\left(e_{5}\right)=\left\{\left(H_{1}, 0.10\right),\left(H_{2}, 0.20\right),\left(H_{3}, 0.70\right)\right\}
\end{gathered}
$$

According to expression (7), we obtain the following importance degree of users:

$$
w_{1}=0.18 ; w_{2}=0.20 ; w_{3}=0.16 ; w_{4}=0.19 ; w_{5}=0.27
$$

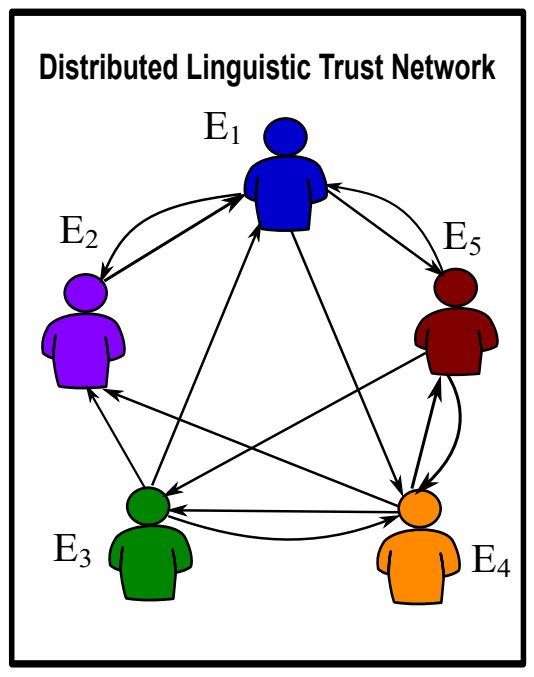

Figure 1: Trust relationship expressed by distributed linguistic in social network

Using the above social network based importance degree $w_{h}$, the individual trust decision matrices can be aggregated as follows: 
Definition 12. Let $\left\{T^{(h)}=\left(t_{i j}^{(h)}\right)_{m \times n} ; h=1,2, \ldots, k\right\}$ be a collection of distributed linguistic trust decision matrices given by a set of users $E=\left\{e_{1}, \ldots, e_{k}\right\}$ under social network. The collective decision matrix guided by trust relationship is $\bar{T}=\left(\bar{t}_{i j}\right)_{m \times n}$ with element

$$
\bar{t}_{i j}=\sum_{h=1}^{k} w_{h} \cdot t_{i j}^{(h)}, i=1, \ldots, m ; j=1, \ldots, n
$$

where $w_{h}$ is given by expression (7).

Example 2. (Example 1 continuation) A group of users $\left\{e_{1}, e_{2}, e_{3}, e_{4}, e_{5}\right\}$ with the following priori trust relationship as depicted in Figure 1 have the same hobby: travel. In a holiday, they want to select a landscape to have a rest, and then they should reach a agreement (consenus) to satisfy every one. After first negation, three are possible travel alternatives: $\left\{x_{1}, x_{2}, x_{3}\right\}$. Five criteria $\left\{c_{1}, c_{2}, c_{3}, c_{4}, c_{5}\right\}$ are considered: Landscape quality; Price; Accommodation; Security and Travel distance, with associated weighting vector $\omega=(0.20,0.40,0.15,0.10,0.15)^{T}$. The group of users give the individual decision making matrices in Tables 2-6.

Table 2: Assessments of three travel alternatives by the user $e_{1}$ based on each criterion

\begin{tabular}{|c|c|c|c|c|c|}
\hline & $C_{1}$ & $C_{2}$ & $C_{3}$ & $C_{4}$ & $C_{5}$ \\
\hline \multirow{3}{*}{$A_{1}$} & $\left(H_{1}, 0.2\right)$, & $\left(H_{1}, 0.6\right)$, & $\left(H_{1}, 0.1\right)$, & $\left(H_{1}, 0.0\right)$, & $\left(H_{1}, 0.2\right)$, \\
\hline & $\left(H_{2}, 0.5\right)$, & $\left(H_{2}, 0.4\right)$ & $\left(H_{2}, 0.2\right)$, & $\left(H_{2}, 0.5\right)$, & $\left(H_{2}, 0.4\right)$, \\
\hline & $\left(H_{3}, 0.3\right)$ & $\left(H_{3}, 0.0\right)$ & $\left(H_{3}, 0.7\right)$ & $\left(H_{3}, 0.5\right)$ & $\left(H_{3}, 0.4\right)$ \\
\hline \multirow{3}{*}{$A_{2}$} & $\left(H_{1}, 0.2\right)$, & $\left(H_{1}, 0.3\right)$, & $\left(H_{1}, 0.1\right)$ & $\left(H_{1}, 0.0\right)$, & $\left(H_{1}, 0.4\right)$, \\
\hline & $\left(H_{2}, 0.4\right)$, & $\left(H_{2}, 0.4\right)$ & $\left(H_{2}, 0.8\right)$, & $\left(H_{2}, 0.7\right)$ & $\left(H_{2}, 0.3\right)$, \\
\hline & $\left(H_{3}, 0.4\right)$ & $\left(H_{3}, 0.3\right)$ & $\left(H_{3}, 0.1\right)$ & $\left(H_{3}, 0.3\right)$ & $\left(H_{3}, 0.3\right)$ \\
\hline \multirow{3}{*}{$A_{3}$} & $\left(H_{1}, 0.1\right)$, & $\left(H_{1}, 0.3\right)$, & $\left(H_{1}, 0.3\right)$, & $\left(H_{1}, 0.2\right)$, & $\left(H_{1}, 0.2\right)$, \\
\hline & $\left(H_{2}, 0.5\right)$, & $\left(H_{2}, 0.7\right)$ & $\left(H_{2}, 0.4\right)$ & $\left(H_{2}, 0.2\right)$, & $\left(H_{2}, 0.8\right)$, \\
\hline & $\left(H_{3}, 0.4\right)$ & $\left(H_{3}, 0.0\right)$ & $\left(H_{3}, 0.3\right)$ & $\left(H_{3}, 0.6\right)$ & $\left(H_{3}, 0.0\right)$ \\
\hline
\end{tabular}

According to the importance degrees of users $W=\{0.18,0.20,0.16,0.19,0.27\}^{T}$, the collective decision matrix $\bar{T}$ are aggregated in Table 7 :

\section{Consensus Model with Optimal Feedback Mechanism under DLTFs}

In order to obtain a collective decision making matrix, the weights associated to each user need to be determined. In traditional GDM models, the weights of user are usually assumed to be known beforehand [13, 16, 50,54]. However, this assumption may be unrealistic or improbable in some cases. In our group decision making under social network environment (SN-GDM), trust relationship between users in a group can be considered as a reliable information source to assign users' weights. 
Table 3: Assessments of three travel alternatives by the user $e_{2}$ based on each criterion

\begin{tabular}{|c|c|c|c|c|c|}
\hline & $C_{1}$ & $C_{2}$ & $C_{3}$ & $C_{4}$ & $C_{5}$ \\
\hline \multirow{3}{*}{$A_{1}$} & $\left(H_{1}, 0.1\right)$ & $(H 1,0.0)$, & $\left(H_{1}, 0.5\right)$ & $\left(H_{1}, 0.0\right)$, & $\left(H_{1}, 0.1\right)$ \\
\hline & $\left(H_{2}, 0.2\right)$ & $(H 2,0.6)$, & $\left(H_{2}, 0.5\right)$, & $\left(H_{2}, 0.4\right)$, & $\left(H_{2}, 0.4\right)$ \\
\hline & $\left(H_{3}, 0.7\right)$ & $(H 3,0.4)$ & $\left(H_{3}, 0\right)$ & $\left(H_{3}, 0.6\right)$ & $\left(H_{3}, 0.5\right)$ \\
\hline \multirow{3}{*}{$A_{2}$} & $\left(H_{1}, 0.4\right)$ & $\left(H_{1}, 0.0\right)$, & $\left(H_{1}, 0.1\right)$, & $\left(H_{1}, 0.4\right)$, & $\left(H_{1}, 0.2\right)$, \\
\hline & $\left(H_{2}, 0.5\right)$ & $\left(H_{2}, 0.3\right)$, & $\left(H_{2}, 0.5\right)$, & $\left(H_{2}, 0.6\right)$, & $\left(H_{2}, 0.4\right)$ \\
\hline & $\left(H_{3}, 0.1\right)$ & $\left(H_{3}, 0.7\right)$ & $\left(H_{3}, 0.4\right)$ & $\left(H_{3}, 0.0\right)$ & $\left(H_{3}, 0.4\right)$ \\
\hline \multirow{3}{*}{$A_{3}$} & $\left(H_{1}, 0.1\right)$ & $\left(H_{1}, 0.0\right)$, & $\left(H_{1}, 0.1\right)$, & $\left(H_{1}, 0.3\right)$, & $\left(H_{1}, 0.0\right)$ \\
\hline & $\left(H_{2}, 0.7\right)$, & $\left(H_{2}, 0.5\right)$, & $\left(H_{2}, 0.2\right)$, & $\left(H_{2}, 0.7\right)$, & $\left(H_{2}, 0.3\right)$ \\
\hline & $\left(H_{3}, 0.2\right)$ & $\left(H_{3}, 0.5\right)$ & $\left(H_{3}, 0.7\right)$ & $\left(H_{3}, 0.0\right)$ & $\left(H_{3}, 0.7\right)$ \\
\hline
\end{tabular}

Table 4: Assessments of three travel alternatives by the user $e_{3}$ based on each criterion

\begin{tabular}{|c|c|c|c|c|c|}
\hline & $C_{1}$ & $C_{2}$ & $C_{3}$ & $C_{4}$ & $C_{5}$ \\
\hline \multirow{3}{*}{$A_{1}$} & $\left(H_{1}, 0.0\right)$, & $\left(H_{1}, 0.3\right)$ & $\left(H_{1}, 0.2\right)$, & $\left(H_{1}, 0.2\right)$ & $\left(H_{1}, 0.2\right)$, \\
\hline & $\left(H_{2}, 0.3\right)$, & $\left(H_{2}, 0.7\right)$ & $\left(H_{2}, 0.1\right)$, & $\left(H_{2}, 0.0\right)$, & $\left(H_{2}, 0.5\right)$, \\
\hline & $\left(H_{3}, 0.7\right)$ & $\left(H_{3}, 0.0\right)$ & $\left(H_{3}, 0.7\right)$ & $\left(H_{3}, 0.8\right)$ & $\left(H_{3}, 0.3\right)$ \\
\hline \multirow{3}{*}{$A_{2}$} & $\left(H_{1}, 0.1\right)$, & $\left(H_{1}, 0.4\right)$ & $\left(H_{1}, 0.8\right)$, & $\left(H_{1}, 0.0\right)$, & $\left(H_{1}, 0.2\right)$, \\
\hline & $\left(H_{2}, 0.2\right)$, & $\left(H_{2}, 0.6\right)$ & $\left(H_{2}, 0.0\right)$, & $\left(H_{2}, 0.1\right)$ & $\left(H_{2}, 0.4\right)$, \\
\hline & $\left(H_{3}, 0.7\right)$ & $\left(H_{3}, 0.0\right)$ & $\left(H_{3}, 0.2\right)$, & $\left(H_{3}, 0.9\right)$ & $\left(H_{3}, 0.4\right)$ \\
\hline \multirow{3}{*}{$A_{3}$} & $\left(H_{1}, 0.0\right)$, & $\left(H_{1}, 0.6\right)$, & $\left(H_{1}, 0.1\right)$, & $\left(H_{1}, 0.1\right)$, & $\left(H_{1}, 0.2\right)$, \\
\hline & $\left(H_{2}, 0.2\right)$ & $\left(H_{2}, 0.0\right)$ & $\left(H_{2}, 0.2\right)$, & $\left(H_{2}, 0.7\right)$ & $\left(H_{2}, 0.0\right)$, \\
\hline & $\left(H_{3}, 0.8\right)$ & $\left(H_{3}, 0.4\right)$ & $\left(H_{3}, 0.7\right)$ & $\left(H_{3}, 0.2\right)$ & $\left(H_{3}, 0.8\right)$ \\
\hline
\end{tabular}


Table 5: Assessments of three travel alternatives by the user $e_{4}$ based on each criterion

\begin{tabular}{|c|c|c|c|c|c|}
\hline & $C_{1}$ & $C_{2}$ & $C_{3}$ & $C_{4}$ & $C_{5}$ \\
\hline \multirow{3}{*}{$A_{1}$} & $\left(H_{1}, 0.1\right)$, & $\left(H_{1}, 0.3\right)$, & $\left(H_{1}, 0.5\right)$, & $\left(H_{1}, 0.4\right)$, & $\left(H_{1}, 0.1\right)$, \\
\hline & $\left(H_{2}, 0.9\right)$, & $\left(H_{2}, 0.5\right)$ & $\left(H_{2}, 0.5\right)$, & $\left(H_{2}, 0.0\right)$, & $\left(H_{2}, 0.4\right)$, \\
\hline & $\left(H_{3}, 0.0\right)$ & $\left(H_{3}, 0.2\right)$ & $\left(H_{3}, 0.0\right)$ & $\left(H_{3}, 0.6\right)$ & $\left(H_{3}, 0.5\right)$ \\
\hline \multirow{3}{*}{$A_{2}$} & $\left(H_{1}, 0.1\right)$, & $\left(H_{1}, 0.2\right)$, & $\left(H_{1}, 0.0\right)$, & $\left(H_{1}, 0.2\right)$, & $\left(H_{1}, 0.3\right)$, \\
\hline & $\left(H_{2}, 0.1\right)$, & $\left(H_{2}, 0.1\right)$ & $\left(H_{2}, 0.6\right)$, & $\left(H_{2}, 0.8\right)$ & $\left(H_{2}, 0.3\right)$, \\
\hline & $\left(H_{3}, 0.8\right)$ & $\left(H_{3}, 0.7\right)$ & $\left(H_{3}, 0.4\right)$, & $\left(H_{3}, 0.0\right)$ & $\left(H_{3}, 0.4\right)$ \\
\hline \multirow{3}{*}{$A_{3}$} & $\left(H_{1}, 0.2\right)$, & $\left(H_{1}, 0.0\right)$, & $\left(H_{1}, 0.2\right)$, & $\left(H_{1}, 0.2\right)$ & $\left(H_{1}, 0.1\right)$, \\
\hline & $\left(H_{2}, 0.5\right)$ & $\left(H_{2}, 0.9\right)$, & $\left(H_{2}, 0.8\right)$, & $\left(H_{2}, 0.4\right)$, & $\left(H_{2}, 0.9\right)$, \\
\hline & $\left(H_{3}, 0.3\right)$ & $\left(H_{3}, 0.1\right)$ & $\left(H_{3}, 0.0\right)$ & $\left(H_{3}, 0.4\right)$ & $\left(H_{3}, 0.0\right)$ \\
\hline
\end{tabular}

Table 6: Assessments of three travel alternatives by the user $e_{5}$ based on each criterion

\begin{tabular}{|c|c|c|c|c|c|}
\hline & $C_{1}$ & $C_{2}$ & $C_{3}$ & $C_{4}$ & $C_{5}$ \\
\hline \multirow{3}{*}{$A_{1}$} & $\left(H_{1}, 0.1\right)$ & $\left(H_{1}, 0.3\right)$ & $\left(H_{1}, 0.6\right)$ & $\left(H_{1}, 0.5\right)$ & $\left(H_{1}, 0.0\right)$, \\
\hline & $\left(H_{2}, 0.1\right)$ & $\left(H_{2}, 0.7\right)$ & $\left(H_{2}, 0.3\right)$ & $\left(H_{2}, 0.0\right)$, & $\left(H_{2}, 0.5\right)$, \\
\hline & $\left(H_{3}, 0.8\right)$ & $\left(H_{3}, 0.0\right)$ & $\left(H_{3}, 0.1\right)$ & $\left(H_{3}, 0.5\right)$ & $\left(H_{3}, 0.5\right)$ \\
\hline \multirow{3}{*}{$A_{2}$} & $\left(H_{1}, 0.0\right)$ & $\left(H_{1}, 0.0\right)$ & $\left(H_{1}, 0.3\right)$ & $\left(H_{1}, 0.4\right)$ & $\left(H_{1}, 0.2\right)$, \\
\hline & $\left(H_{2}, 0.1\right)$ & $\left(H_{2}, 0.3\right)$ & $\left(H_{2}, 0.1\right)$ & $\left(H_{2}, 0.6\right)$ & $\left(H_{2}, 0.5\right)$, \\
\hline & $\left(H_{3}, 0.9\right)$ & $\left(H_{3}, 0.7\right)$ & $\left(H_{3}, 0.6\right)$ & $\left(H_{3}, 0.0\right)$ & $\left(H_{3}, 0.3\right)$ \\
\hline \multirow{3}{*}{$A_{3}$} & $\left(H_{1}, 0.3\right)$ & $\left(H_{1}, 0.1\right)$ & $\left(H_{1}, 0.0\right)$, & $\left(H_{1}, 0.2\right)$, & $\left(H_{1}, 0.1\right)$, \\
\hline & $\left(H_{2}, 0.7\right)$ & $\left(H_{2}, 0.8\right)$ & $\left(H_{2}, 0.7\right)$ & $\left(H_{2}, 0.7\right)$ & $\left(H_{2}, 0.3\right)$, \\
\hline & $\left(H_{3}, 0.0\right)$ & $\left(H_{3}, 0.1\right)$ & $\left(H_{3}, 0.3\right)$ & $\left(H_{3}, 0.1\right)$ & $\left(H_{3}, 0.6\right)$ \\
\hline
\end{tabular}




\begin{tabular}{|c|c|c|c|c|c|}
\hline & $C_{1}$ & $C_{2}$ & $C_{3}$ & $C_{4}$ & $C_{5}$ \\
\hline \multirow{3}{*}{$A_{1}$} & $\left(H_{1}, 0.10\right)$ & $\left(H_{1}, 0.29\right)$ & $\left(H_{1}, 0.41\right)$ & $\left(H_{1}, 0.24\right)$ & $\left(H_{1}, 0.11\right)$ \\
\hline & $\left(H_{2}, 0.38\right)$ & $\left(H_{2}, 0.59\right)$ & $\left(H_{2}, 0.33\right)$ & $\left(H_{2}, 0.17\right)$ & $\left(H_{2}, 0.44\right)$, \\
\hline & $\left(H_{3}, 0.52\right)$ & $\left(H_{3}, 0.12\right)$ & $\left(H_{3}, 0.26\right)$ & $\left(H_{3}, 0.59\right)$ & $\left(H_{3}, 0.45\right)$ \\
\hline \multirow{3}{*}{$A_{2}$} & $\left(H_{1}, 0.15\right)$ & $\left(H_{1}, 0.16\right)$ & $\left(H_{1}, 0.25\right)$ & $\left(H_{1}, 0.23\right)$ & $\left(H_{1}, 0.25\right)$ \\
\hline & $\left(H_{2}, 0.25\right)$ & $\left(H_{2}, 0.33\right)$ & $\left(H_{2}, 0.39\right)$ & $\left(H_{2}, 0.58\right)$ & $\left(H_{2}, 0.39\right)$, \\
\hline & $\left(H_{3}, 0.60\right)$ & $\left(H_{3}, 0.51\right)$ & $\left(H_{3}, 0.36\right)$, & $\left(H_{3}, 0.19\right)$ & $\left(H_{3}, 0.36\right)$ \\
\hline \multirow{3}{*}{$A_{3}$} & $\left(H_{1}, 0.16\right)$ & $\left(H_{1}, 0.18\right)$ & $\left(H_{1}, 0.13\right)$ & $\left(H_{1}, 0.20\right)$ & $\left(H_{1}, 0.11\right)$ \\
\hline & $\left(H_{2}, 0.55\right)$ & $\left(H_{2}, 0.61\right)$ & $\left(H_{2}, 0.48\right)$ & $\left(H_{2}, 0.55\right)$ & $\left(H_{2}, 0.45\right)$, \\
\hline & $\left(H_{3}, 0.29\right)$ & $\left(H_{3}, 0.21\right)$ & $\left(H_{3}, 0.39\right)$ & $\left(H_{3}, 0.25\right)$ & $\left(H_{3}, 0.44\right)$ \\
\hline
\end{tabular}

Once the trust based users' weights are determined and the collective decision matrix is calculated, the consensus degree (CD) at three levels for each user is expressed as: (1) elements level; (2) alternatives level; and (3) decision matrix level. When each user reaches a set threshold value of consensus degree, the resolution process of the SN-GDM is carried out; otherwise the inconsistent users are identified and then a feedback mechanism is activated to produce recommendation advises for them to higher consensus degree. However, the existed feedback mechanisms are usually assumed a fixed a feedback parameter beforehand, and then the inconsistent users are forced to implement the recommendation advices given by the feedback mechanism. Therefore, they do not know how much cost should afford if they want to reach consensus. To resolve this issue, this article will investigate a minimum adjustment cost feedback mechanism in which an optimal model is proposed to determine the boundary feedback parameter. Based on this boundary feedback parameter information, the inconsistent users are able to know how to adopt the recommendation advices if they are willing to reach the threshold value of group consensus degree.

The minimum adjustment cost feedback mechanism for consensus in SN-GDM with distributed linguistic trust is depicted in Figure 2. In detail, it has the following five steps:

(1) Introducing the definition of Distributed Linguistic Trust;

(2) Building Trust Relationship and determining weights of users;

(3) Computing consensus indexes at three levels;

(4) Minimum adjustment cost feedback mechanism; and

(5) Selection Process. 


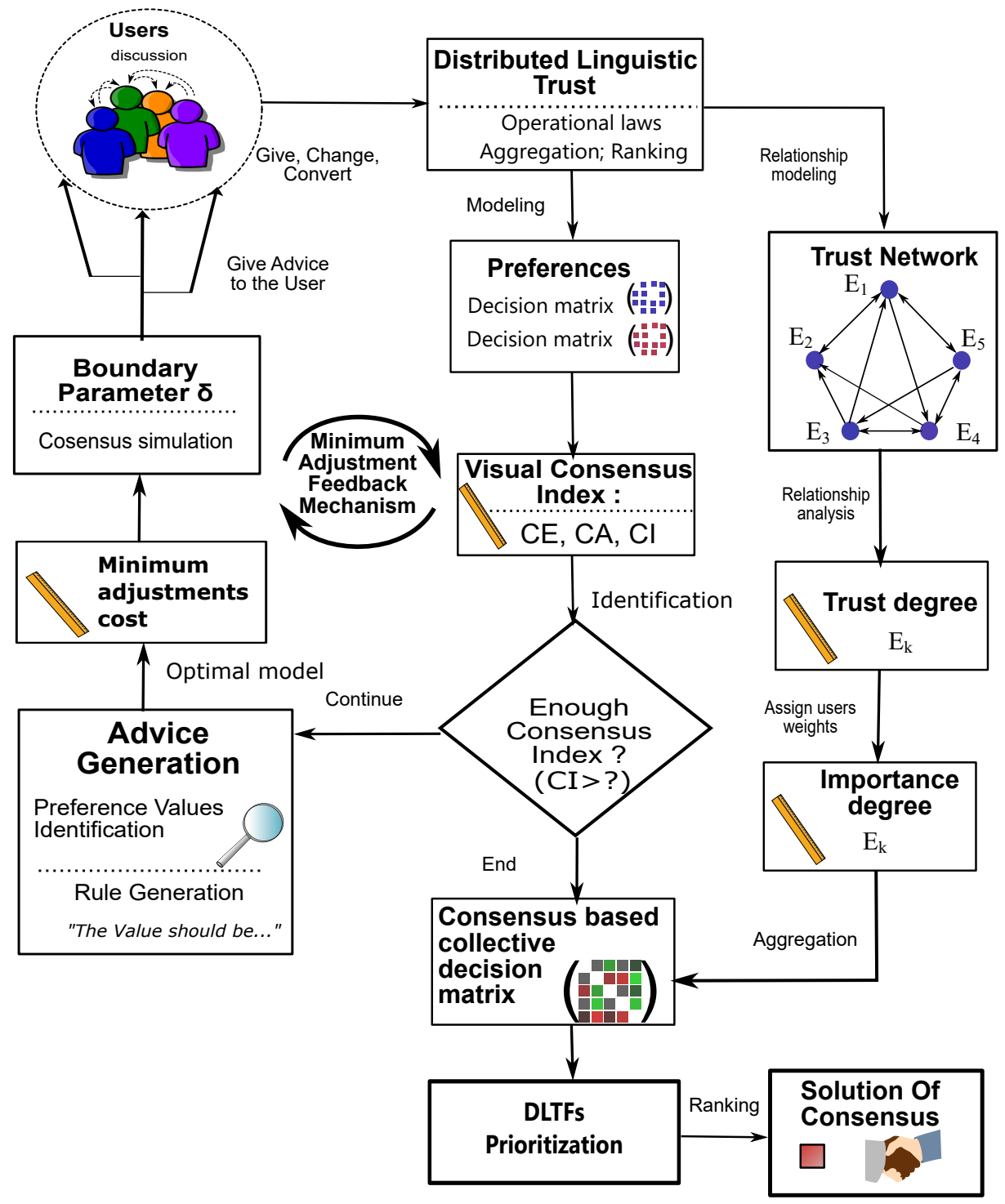

Figure 2: Minimum adjustment cost feedback mechanism for consensus in SN-GDM with DLT

The first and second steps have already been introduced in Section 2 and Section 3, respectively. The others are going to be presented in the following subsections.

\subsection{Three Levels of Consensus Indexes}

Let $\widetilde{T}^{h}=\left(\widetilde{t}_{i j}^{h}\right)_{n \times n}$ and $\widetilde{T}^{l}=\left(\widetilde{t}_{i j}\right)_{n \times n}$ be the decision matrices with distributed linguistic trust information provided by users $e_{h}$ and $e_{l}$, respectively. A distance function $(d)$ between distributed linguistic trust function is used to define the consensus index between these users as follows:

The consensus index of an user with the group at the three different levels of a relation are defined next:

Level 1. Consensus index at evaluation element levels. The consensus index of an user $e_{h}$ with respect 
to the group on the alternatives $x_{i}$ under criterion $c_{j}$ is:

$$
C E_{i j}^{h}=1-d\left(t_{i j}^{h}, \bar{t}_{i j}\right)=1-\frac{1}{\xi} \sum_{\alpha=1}^{\xi}\left|\left(\varphi_{\alpha}\right)_{i j}^{h}-\left(\bar{\varphi}_{\alpha}\right)_{i j}\right|
$$

Level 2. Consensus index at alternatives level. The consensus index of an user $e_{h}$ with respect to the group on the alternative $x_{i}$ is:

$$
C A_{i}^{h}=\frac{1}{n} \sum_{j=1}^{n} C E_{i j}^{h}
$$

Level 3. Consensus index at trust decision matrix level. The consensus index of an user $e_{h}$ to the group on decision matrix is:

$$
C I^{h}=\frac{1}{m} \sum_{i=1}^{m} C A_{i}^{h}
$$

Example 3. (Example 3(b) continuation) The consensus indexes on alternatives level are:

$$
\begin{gathered}
C E^{1}=\left(\begin{array}{ccccc}
0.85 & 0.79 & 0.71 & 0.78 & 0.94 \\
0.87 & 0.86 & 0.73 & 0.85 & 0.90 \\
0.93 & 0.86 & 0.89 & 0.77 & 0.71
\end{array}\right) \quad C E^{2}=\left(\begin{array}{llllll}
0.88 & 0.81 & 0.83 & 0.84 & 0.97 \\
0.67 & 0.87 & 0.90 & 0.87 & 0.97 \\
0.90 & 0.81 & 0.79 & 0.83 & 0.83
\end{array}\right) \\
C E^{3}=\left(\begin{array}{lllll}
0.88 & 0.92 & 0.71 & 0.86 & 0.90 \\
0.93 & 0.66 & 0.63 & 0.53 & 0.97 \\
0.66 & 0.59 & 0.79 & 0.90 & 0.70
\end{array}\right) \quad C E^{4}=\left(\begin{array}{llllll}
0.65 & 0.94 & 0.83 & 0.89 & 0.97 \\
0.87 & 0.85 & 0.83 & 0.85 & 0.94 \\
0.97 & 0.81 & 0.74 & 0.90 & 0.70
\end{array}\right) \\
C E^{5}=\left(\begin{array}{ccccc}
0.81 & 0.92 & 0.87 & 0.83 & 0.93 \\
0.80 & 0.87 & 0.81 & 0.87 & 0.93 \\
0.81 & 0.87 & 0.85 & 0.90 & 0.89
\end{array}\right)
\end{gathered}
$$

The consensus indexes on alternatives level are:

$$
\begin{array}{ll}
C A^{1}=(0.81,0.84,0.83) ; & C A^{2}=(0.87,0.86,0.83) ; \\
C A^{3}=(0.85,0.74,0.73) ; & C A^{4}=(0.86,0.87,0.82) ; \\
C A^{5}=(0.87,0.86,0.86) . &
\end{array}
$$

The individual consensus indexes at the decision matrix level are:

$$
C I^{1}=0.83, C I^{2}=0.85, C I^{3}=0.77, C I^{4}=0.85, C I^{5}=0.86 .
$$

Notice that consensus is defined as the full and unanimous agreement of all the users regarding all the feasible alternatives. However, the chances for reaching such a full agreement are rather low, which means the threshold value of consensus $\gamma<1$. Also in most cases, if more than half of people achieve consensus, the decision-making result may be acceptable. Therefore, threshold value should be $\gamma \in[0.5,1)$. In this article, we suppose the threshold value of $\gamma=0.8$. Since $C I^{3}<\gamma$, then user $e_{3}$ is inconsistent with a set threshold value of consenus in Figure 3. Hence, the feedback mechanism is activated to help $e_{3}$ to modify his/her inconsistent evaluation elements to higher consensus status. 

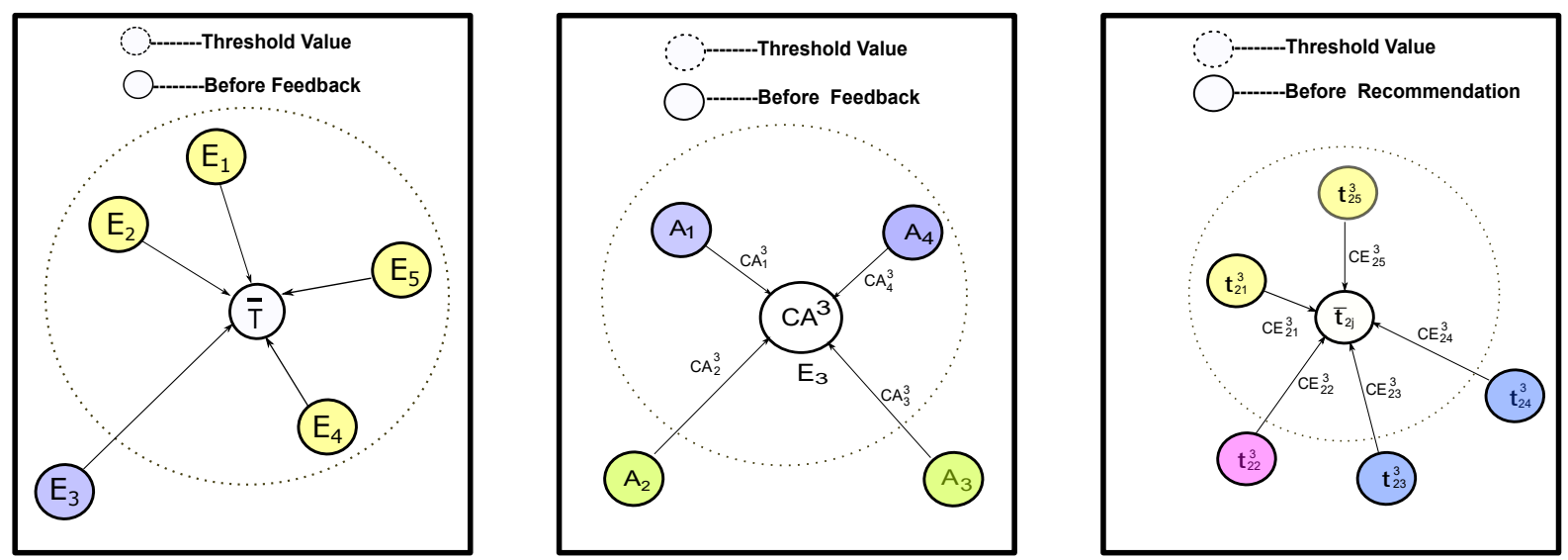

(a) The visual consensus index at the decision matrix level: alternatives level for user $e_{3}$ (b) The visual consensus index at the element $A_{2}$ level for user $e_{3}$

Figure 3: Three visual levels of consensus before feedback mechanism

\subsection{Minimum Adjustment Cost Feedback Mechanism}

The minimum adjustment cost feedback mechanism including three steps: (1)Identification of the inconsistent evaluation elements with distributed linguistic trust information, (2)Generation of recommended advices with boundary feedback parameter, and (3) Group consensus convergence analysis, which are described in detail below:

(1) Identification of the inconsistent evaluation elements: The set of evaluation elements by distribute linguistic trust information that less than the threshold value of consensus level is identified as follows:

Step 1. Users with a consensus index at decision matrix lower than the threshold value $\gamma$ are identified:

$$
E X P C H=\left\{h \mid C I^{h}<\gamma\right\}
$$

Step 2. For the identified users in Step 1, their alternatives with a consensus index $C A_{i}^{h}$ lower than the threshold $\gamma$ are identified:

$$
A L T=\left\{(h, i) \mid h \in E X P C H \wedge C A_{i}^{h}<\gamma\right\}
$$

Step 3. Finally, the evaluation elements to be replaced are those with a consensus index $C E_{i}^{h}$ under the threshold $\gamma$ :

$$
A P S=\left\{(h, i, j) \mid(h, i) \in A L T \wedge C E_{i j}^{h}<\gamma\right\}
$$

Example 4. (Example 3 continuation). Evaluation elements with distributed linguistic trust information to Change. The set of 3-tuples APS identified under the threshold $\gamma$ 
are:

$$
A P S=\{(3,2,2),(3,2,3),(3,2,4),(3,3,1),(3,3,2),(3,3,3),(3,3,5)\}
$$

(2) Generation of recommendation advices with boundary feedback parameter: The feedback mechanism generates advices to the inconsistent users and for the preference values previously identified in $A P S$ containing the new preference values for a higher consensus state.

For all $(h, i, k) \in A P S$, the following rule is feed-backed to the corresponding user in:

"Change your evaluation elements for the pair of alternatives $(i, j)$ to a value closer to $r t_{i j}^{h}$ :"

$$
r t_{i j}^{h}=(1-\delta) \cdot t_{i j}^{h}+\delta \cdot \bar{t}_{i j},
$$

where $\delta \in[0,1]$ is a feedback mechanism parameter to control the accepting degree of recommendation advices, and $\bar{t}_{i j}$ is calculated by expression (8).

Obviously, when the feedback parameter $\delta$ takes value 1 , the original assessment is completely replaced by the collective assessment, while when $\delta$ takes value 0 the original assessment is kept unchanged. The more the feedback parameter $\delta_{\text {min }}$, the higher the adjustment costs. Therefore, how to select a boundary parameter is one important issue in interaction of group decision making. However, the existed feedback mechanisms neglect this issue in which the parameter $\delta$ is fixed beforehand [5, 21, 42].

Recently, the minimum adjustment cost role for consensus is proposed by Dong et.al $[14,15]$, which produces the reaccommodated values to the satisfied consensus level. Inspired by this reasonable adjustment rule in interaction of group users, we shall build an optimized model to determine the boundary parameter $\delta$, and then users can achieve an acceptable compromise between group consensus with minimum costs. To do that, we first show how the consensus index at the elements level $C T E_{i j}^{h}$ is affected by the feedback parameter $\delta$ when advices are adopted in the following Proposition similar in [44].

Proposition 1. If only user $e_{h}$ adopts recommendations advices then the new consensus index at element level for such user will be greater or equal than his/her previous consensus index at element level. Furthermore, the new consensus index at element level is monotonic increasing with respect to parameter $\delta$.

Proof. After the user $e_{h}$ adopts recommended value for alternative $x_{i}$ under criteria $c_{j}$, then assessment values for alternative $x_{i}$ under criteria $c_{j}$ are divided into two groups: the new preference value $\left\{r t_{i j}^{h} \mid r t_{i j}^{h}=(1-\delta) t_{i j}^{h}+\delta \times \bar{t}_{i j}\right\}$ and the set of unchanged assessment values $\left\{\left(r t_{i j}^{s}\right) \mid r t_{i j}^{s}=t_{i j}^{s}, s=1, \cdots, k, s \neq h\right\}$. Then, the collective decision making matrix of next round 
is calculated as:

$$
\overline{r t}_{i j}=w_{T}^{h} \cdot r t_{i j}^{h}+\sum_{s=1, s \neq h}^{k} w_{T}^{s} \cdot r t_{i j}^{s}=w_{T}^{h} \cdot r t_{i j}^{h}-w_{T}^{h} \cdot t_{i j}^{h}+\bar{t}_{i j}
$$

and the new consensus index for alternative $x_{i}$ under criteria $c_{j}$, which we will denote by $\overline{C E}_{i j}^{h}$ to differentiate it from the previous one $C E_{i j}^{h}$, would be

$$
\begin{aligned}
\overline{C E}_{i j}^{h} & =1-\left|r t_{i j}^{h}-\overline{r t}_{i j}\right| \\
& =1-\left|(1-\delta) \cdot t_{i j}^{h}+\delta \cdot \bar{t}_{i j}-\left(w_{T}^{h} \cdot r t_{i j}^{h}-w_{T}^{h} \cdot t_{i j}^{h}+\bar{t}_{i j}\right)\right| \\
& =1-\left|(1-\delta) \cdot t_{i j}^{h}+\delta \cdot \bar{t}_{i j}-\left(w_{T}^{h} \cdot\left((1-\delta) \cdot t_{i j}^{h}+\delta \bar{t}_{i j}\right)-w_{T}^{h} \cdot t_{i j}^{h}+\bar{t}_{i j}\right)\right| \\
& =1+\left(\delta \cdot\left(1-w_{T}^{h}\right)-1\right) \cdot\left|t_{i j}^{h}-\bar{t}_{i j}\right| \\
& =\delta \cdot\left(1-w_{T}^{h}\right) \cdot\left|t_{i j}^{h}-\bar{t}_{i j}\right|+C E_{i j}^{h}
\end{aligned}
$$

Because $0 \leq\left(1-w_{T}^{h}\right) \leq 1$, and $\delta \in[0,1]$, then consequently the next current consensus index $\overline{C E}_{i j}^{h}$ is monotonic increasing with respect to parameter $\delta$. When parameter $\delta=0$, user $e_{h}$ dose not adopt the recommendation advices, and then the new consensus index $\overline{C E}_{i j}^{h}$ is equal to the previous consensus index $C E_{i j}^{h}$.

From Proposition (1), it is clearly that: the more the parameter $\delta$, the new consensus degree of user $e_{h}$. However, apart from consensus, decision makers intend to keep their individuals original opinions because that more adoption need more adjust cost $[9,19,52]$. Therefore, decision makers need keep balance between consensus degree and adjustment cost. In other words, they are willing to reach the threshold of consensus degree with lower adjustment cost for adopting recommendation advices. Therefore, we need determine the boundary parameter $\delta_{\text {min }}$.

Let the original decision making matrices are divided into two groups: the most inconsistent decision matrix $T^{h}$ and other decision matrices $T^{s}$. After the user $e_{h}$ adopts the recommendation advices, we obtain that $\left\{R T^{h}=\left(r t_{i j}^{h}\right) \mid r t_{i j}^{h}=(1-\delta) t_{i j}^{h}+\delta \bar{t}_{i j}^{h}, i j \in A P S ; r t_{i j}^{h}=t_{i j}^{h}, i j \notin A P S\right\}$ be the new decision making matrix after adopting, and $\left\{R T^{s}=T^{s}=\left(t_{i j}^{s}\right), s=1, \ldots, k, s \neq h\right\}$ be the set of unchanged decision making matrix. Then, the adjustments cost of the most inconsistent decision matrix $T^{h}$ can be defined as follows.

Definition 13. After the most inconsistent user accepts the recommendation advices, the adjustments cost of feedback can be calculated as:

$$
F=\left|T^{h}-R T^{h}\right|
$$

Proposition 2. The adjustments cost function $F$ is monotonic increasing with respect to parameter $\delta$. 
Proof.

$$
\begin{aligned}
F & =\left|T^{h}-R T^{h}\right| \\
& =\sum_{h, i, j \in A P S}\left|t_{i j}^{h}-\left((1-\delta) t_{i j}^{h}+\delta \bar{t}_{i j}^{h}\right)\right| \\
& =\sum_{h, i, j \in A P S} \delta\left|t_{i j}^{h}-\bar{t}_{i j}^{h}\right|
\end{aligned}
$$

Hence, the optimal model of minimum adjustments established as:

$$
\begin{aligned}
& \operatorname{Min}\left|T^{h}-R T^{h}\right| \\
& \text { s.t. }\left\{\begin{array}{l}
C I^{h}\left(R T^{h}, \overline{R T}\right) \geq \gamma \\
C I^{s}\left(R T^{s}, \overline{R T}\right) \geq \gamma, s=1, \ldots, k, s \neq h \\
\overline{R T}=D T W A\left(R T^{h}, R T^{1}, \ldots R T^{s}, \ldots, R T^{k}\right) \\
0 \leq \gamma \leq 1
\end{array}\right.
\end{aligned}
$$

Also, the above optimal model can be rewritten as:

$$
\begin{aligned}
& \operatorname{Min} \sum_{h, i, j \in A P S} \delta\left|t^{h}-\bar{t}_{i j}^{h}\right| \\
& \text { s.t. }\left\{\begin{array}{l}
C I^{h}\left(R T^{h}, \overline{R T}\right) \geq \gamma \\
C I^{s}\left(R T^{s}, \overline{R T}\right) \geq \gamma, s=1, \ldots, k, s \neq h \\
\overline{R T}=D T W A\left(R T^{h}, R T^{1}, \ldots R T^{s}, \ldots, R T^{k}\right)
\end{array}\right.
\end{aligned}
$$

By resolve model (15), we can determine the boundary feedback parameter $\delta_{\text {min }}$, and then, from Proposition 2, the inconsistent user $e^{h}$ affords the minimum adjustments cost.

Example 5. (Example 4 continuation). Determining the boundary feedback parameter. With the supposed threshold value $\gamma=0.8$, the above model (15) can be transformed to the following optimal model (16).

$$
\begin{aligned}
& \operatorname{Min} \sum_{h, i, j \in A P S} \delta\left|t^{h}-\bar{t}_{i j}^{h}\right| \\
& \text { s.t. }\left\{\begin{array}{l}
C I^{h}\left(R T^{h}, \overline{R T}\right) \geq 0.8 \\
C I^{s}\left(R T^{s}, \overline{R T}\right) \geq 0.8, s=1, \ldots, k, s \neq h \\
\overline{R T}=D T W A\left(R T^{h}, R T^{1}, \ldots R T^{s}, \ldots, R T^{k}\right)
\end{array}\right.
\end{aligned}
$$

By resolving the optimal model (16), we can get the boundary feedback parameter $\delta_{\min }=0.18$.

Example 6. (Example 5 continuation). Generation of Advice. Taking a value of $\delta_{\min }=0.18$, the recommendations for user $e_{3}$ are:

- Your trust assessment of alternative $x_{2}$ under criteria $C_{2}$ should be updated closely to $\left\{\left(H_{1}, 0.36\right),\left(H_{2}, 0.55\right),\left(H_{3}, 0.09\right)\right\}$. 


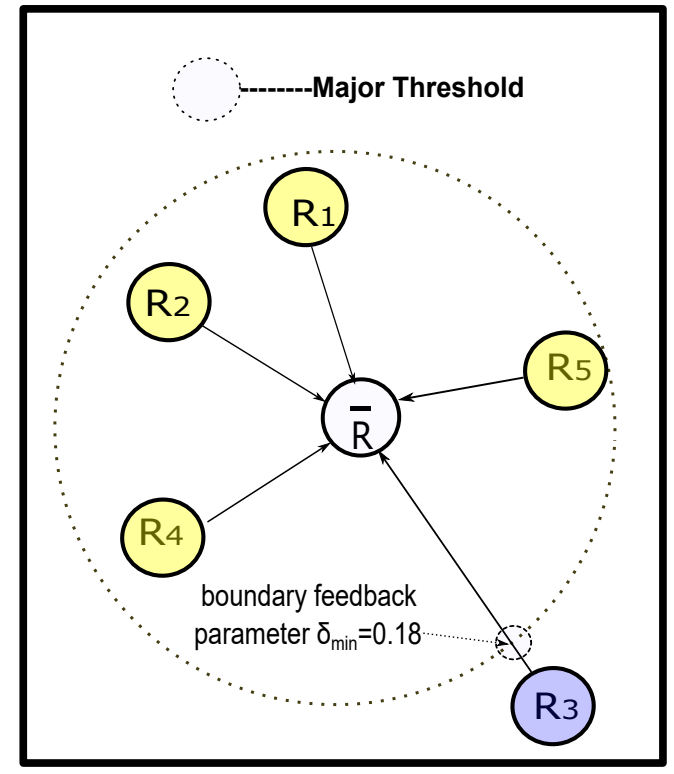

Figure 4: Determine the boundary feedback parameter $\delta_{\min }$ by optimal model

- Your trust assessment of alternative $x_{2}$ under criteria $C_{3}$ should be updated closely to $\left\{\left(H_{1}, 0.70\right),\left(H_{2}, 0.07\right),\left(H_{3}, 0.23\right)\right\}$.

- Your trust assessment of alternative $x_{2}$ under criteria $C_{4}$ should be updated closely to $\left\{\left(H_{1}, 0.04\right),\left(H_{2}, 0.19\right),\left(H_{3}, 0.77\right)\right\}$.

- Your trust assessment of alternative $x_{3}$ under criteria $C_{1}$ should be updated closely to $\left\{\left(H_{1}, 0.03\right),\left(H_{2}, 0.26\right),\left(H_{3}, 0.71\right)\right\}$.

- Your trust assessment of alternative $x_{3}$ under criteria $C_{2}$ should be updated closely to $\left\{\left(H_{1}, 0.52\right),\left(H_{2}, 0.11\right),\left(H_{3}, 0.37\right)\right\}$.

- Your trust assessment of alternative $x_{3}$ under criteria $C_{3}$ should be updated closely to $\left\{\left(H_{1}, 0.11\right),\left(H_{2}, 0.25\right),\left(H_{3}, 0.64\right)\right\}$.

- Your trust assessment of alternative $x_{3}$ under criteria $C_{5}$ should be updated closely to $\left\{\left(H_{1}, 0.19\right),\left(H_{2}, 0.09\right),\left(H_{3}, 0.72\right)\right\}$.

After user $e_{3}$ adopts the recommendation advices, the new collective decision making matrix with 
distributed linguistic trust would be

$$
\overline{R T}=\left\{\begin{array}{r}
\left\{\begin{array}{l}
\left(H_{1}, 0.10\right), \\
\left(H_{2}, 0.38\right), \\
\left(H_{3}, 0.52\right)
\end{array}\right\}\left\{\begin{array}{l}
\left(H_{1}, 0.29\right), \\
\left(H_{2}, 0.59\right), \\
\left(H_{3}, 0.12\right)
\end{array}\right\}\left\{\begin{array}{l}
\left(H_{1}, 0.41\right), \\
\left(H_{2}, 0.33\right), \\
\left(H_{3}, 0.26\right)
\end{array}\right\}\left\{\begin{array}{l}
\left(H_{1}, 0.24\right), \\
\left(H_{2}, 0.17\right), \\
\left(H_{3}, 0.59\right)
\end{array}\right\}\left\{\begin{array}{l}
\left(H_{1}, 0.11\right), \\
\left(H_{2}, 0.44\right), \\
\left(H_{3}, 0.45\right)
\end{array}\right\} \\
\left\{\begin{array}{l}
\left(H_{1}, 0.15\right), \\
\left(H_{2}, 0.25\right), \\
\left(H_{3}, 0.60\right)
\end{array}\right\}\left\{\begin{array}{l}
\left(H_{1}, 0.15\right), \\
\left(H_{2}, 0.32\right), \\
\left(H_{3}, 0.53\right)
\end{array}\right\}\left\{\begin{array}{l}
\left(H_{1}, 0.23\right), \\
\left(H_{2}, 0.40\right), \\
\left(H_{3}, 0.37\right)
\end{array}\right\}\left\{\begin{array}{l}
\left(H_{1}, 0.23\right), \\
\left(H_{2}, 0.59\right), \\
\left(H_{3}, 0.18\right)
\end{array}\right\}\left\{\begin{array}{l}
\left(H_{1}, 0.25\right), \\
\left(H_{2}, 0.39\right), \\
\left(H_{3}, 0.36\right)
\end{array}\right\} \\
\left\{\begin{array}{l}
\left(H_{1}, 0.16\right), \\
\left(H_{2}, 0.56\right), \\
\left(H_{3}, 0.28\right)
\end{array}\right\}\left\{\begin{array}{l}
\left(H_{1}, 0.16\right), \\
\left(H_{2}, 0.63\right), \\
\left(H_{3}, 0.21\right)
\end{array}\right\}\left\{\begin{array}{l}
\left(H_{1}, 0.13\right), \\
\left(H_{2}, 0.49\right), \\
\left(H_{3}, 0.38\right)
\end{array}\right\}\left\{\begin{array}{l}
\left(H_{1}, 0.20\right), \\
\left(H_{2}, 0.55\right), \\
\left(H_{3}, 0.25\right)
\end{array}\right\}\left\{\begin{array}{l}
\left(H_{1}, 0.11\right), \\
\left(H_{2}, 0.47\right), \\
\left(H_{3}, 0.42\right)
\end{array}\right\}
\end{array}\right.
$$

The new consensus levels are calculated as:

$$
C I^{1}=0.83, C I^{2}=0.85, C I^{3}=0.80, C I^{4}=0.85, C I^{5}=0.87 .
$$

Since all users are above of the consensus threshold value $\gamma=0.8$, the consensual collective decision matrix is computed for the final solution.

According to expression (13), we also can calculate the minimum adjust cost of feedback with $\delta_{\min }=0.18$ as:

$$
F_{\delta_{\min }}=1.32
$$

However, existing feedback mechanisms $[5,21,41,42]$ are not able to arrive the minimum adjust cost because they usually suppose a subjective feedback parameter beforehand. This subjective feedback parameter is not reasonable in most cases. For example, if we let $\delta=0.5$, we obtain the adjustment cost of feedback as:

$$
F_{\delta=0.5}=3.67
$$

Obviously, this case need afford a higher adjustment cost to reach consensus, and then it may not be adopted in real decision making process.

(3) Group consensus convergence analysis:

To study the status of group consensus in each round of feedback, we have the following proposition.

Proposition 3. If the inconsistent user $e_{h}$ adopts the recommendation advices, then group consensus degree will be greater or equal than the new consensus index of $e_{h}$ at element level.

$$
\overline{C E}_{i j}^{h} \leq C E_{i j}\left(\overline{r t}_{i j}, \bar{t}_{i j}\right)
$$

Furthermore, the group consensus at element level is also monotonic decreasing with respect to parameter $\delta$. 
Proof.

$$
\begin{aligned}
C E_{i j}\left(\overline{r t}_{i j}, \bar{t}_{i j}\right) & =1-\left|w_{T}^{h} r t_{i j}^{h}-w_{T}^{h} t_{i j}^{h}\right| \\
& =1-\left|w_{T}^{h}\left((1-\delta) t_{i j}^{h}+\delta \bar{t}_{i j}\right)-w_{T}^{h} t_{i j}^{h}\right| \\
& =1-w_{T}^{h} \delta\left|t_{i j}^{h}-\bar{t}_{i j}\right|
\end{aligned}
$$

Then, we have

$$
\begin{aligned}
C E_{i j}\left(\overline{r t}_{i j}, \bar{t}_{i j}\right)-C E_{i j}^{h} & =1-w_{T}^{h} \delta\left|t_{i j}^{h}-\bar{t}_{i j}\right|-\left(1+\left(\delta\left(1-w_{T}^{h}\right)-1\right)\left|t_{i j}^{h}-\bar{t}_{i j}\right|\right) \\
& =(1-\delta)\left|t_{i j}^{h}-\bar{t}_{i j}\right| \\
& \geq 0
\end{aligned}
$$

which finishes the proof of Proposition (3).

In our proposed consensus interaction model, Proposition 1 guarantees the new consensus index of $e_{h}$ is improved after adopting the recommendation advices advices. Further, Proposition 3 shows us that the group consensus degree is greater than the new consensus index of $e_{h}$. Therefore, these two propositions can guarantee group consensus convergence.

Example 7. (Finishing) By the associated weighting vector of criteria $\omega=(0.20,0.40,0.15,0.10,0.15)^{T}$, the collective overall evaluation values $(\mathrm{i}=1,2,3)$ of the three alternatives are calculated as:

$$
\begin{aligned}
& \bar{r}_{1}=\left\{\left(H_{1}, 0.24\right),\left(H_{2}, 0.44\right),\left(H_{3}, 0.32\right)\right\}, \\
& \bar{r}_{2}=\left\{\left(H_{1}, 0.19\right),\left(H_{2}, 0.36\right),\left(H_{3}, 0.46\right)\right\}, \\
& \bar{r}_{3}=\left\{\left(H_{1}, 0.15\right),\left(H_{2}, 0.56\right),\left(H_{3}, 0.29\right)\right\} .
\end{aligned}
$$

Their corresponding expected trust scores are:

$$
E\left(\bar{r}_{1}\right)=2.08, E\left(\bar{r}_{2}\right)=2.25, E\left(\bar{r}_{3}\right)=2.13
$$

Then, we have

$$
x_{2} \succ x_{3} \succ x_{1}
$$

Therefore, alternative $x_{2}$ is selected as the group solution of consensus.

\section{Conclusions}

This paper presents a minimum adjustment cost feedback mechanism for higher consensus in social network group decision making under distributed linguistic trust information. It has the following main advantages and differences with respect to other consensus models proposed in the literature: 
(1) It develops the distributed linguistic trust decision space to model uncertainty in GDM including the novel concepts of distributed linguistic trust functions, expectation degree, uncertainty degree, and the ranking order relation for distributed linguistic trust functions. Then, it can express trust opinions with linguistic such as: high, middle and low. Therefore, it is suitable to represent the uncertainty or fuzziness of trust relationship under social network. Hence, the concept of indegree of centrality with distributed linguistic trust for individual user is defined, and it is regarded as a reliable source of importance associated to users in determining their aggregation weights. Consequently, our proposed approach resolves the unrealistic assumption of users' weights to be known beforehand in the traditional GDM methods.

(2) It investigates a minimum adjustment cost feedback mechanism to generate personalised advice for the inconsistent users to select appropriate feedback parameter to reach the threshold value of group consensus degree. To do that, the relationship between feedback parameter and new consensus degree of next ground is explored. An optimal model is established to achieve an acceptable compromise between group consensus with minimum costs. By resolving this model, the boundary feedback parameter $\delta_{\min }$ is determined, which means the cost the inconsistent user should afford to reach the threshold value of group consensus degree.

This proposed minimum adjustment cost feedback mechanism utilizes the weighted average operator of distributed linguistic to aggregate individual opinions to produce the recommendation advices. The trust mechanism is studied in the process of producing recommendation advices and proved that it can reduce the change cost in achieving the threshold value of consensus in [29]. We believe this conclusion can also be used to improve the feedback mechanism in GDM. To do that, our future aim is to develop appropriate propagation operators with distribution linguistic trust to generate complete trust relationship for a social network. Then, a new minimum adjustment cost feedback mechanism with trust recommended advices for consensus in GDM can be established. Furthermore, because group travel is a typical small number of users decision making problem, we also aim in future to develop a consensus method for larger number of users in SN.

\section{Acknowledgements}

The authors are very grateful to the anonymous referees for their valuable comments and suggestions that have helped the authors to improve considerably the quality of this paper. This work was supported by National Natural Science Foundation of China (NSFC) (No.71571166, 71331002), Zhejiang Provincial Xingmiao Talent Foundation of China (No.2017R404001), the grants TIN201340658-P and TIN2016-75850-R support by FEDER funds. 
[1] Alonso, S., Herrera-Viedma, E., Chiclana, F., and Herrera, F. (2010). A web based consensus support system for group decision making problems and incomplete preferences. Information Sciences 180, 4477-4495.

[2] Cabrerizo, F. J, Chiclana, F., Al-Hmouz, R., Morfeq, A., Balamash, A. S. and Herrera-Viedma, E. (2015). Fuzzy decision making and consensus: challenges. Journal of Intelligent $\&$ Fuzzy Systems. 29 (3), 1109-1118.

[3] Cabrerizo, F. J, Herrera-Viedma, E. and Pedrycz, W. (2013). A Method based on PSO and Granular Computing of Linguistic Information to Solve Group Decision Making Problems defined in Heterogeneous Contexts. European Journal of Operational Research 230(3), 624-633.

[4] Castro, J., Lu, J., Zhang, G., Dong, Y. C. and Martínez, L. Opinion dynamics based group recommender systems. IEEE Transactions on Systems, Man and Cybernetics: Systems (In Press), DOI:10.1109/TSMC.2017.2695158.

[5] Cheng, S. M., Cheng, S. H., and Lin, T. E. (2015). Group decision making systems using group recommendations based on interval fuzzy preference relations and consistency matrices. Information Sciences 298, 555-567.

[6] Chiclana, F., Tapia-Garcia, J. M., del Moral, M. J., and Herrera-Viedma, E. (2013). A statistical comparative study of different similarity measures of consensus in group decision making. Information Sciences 221, 110-123.

[7] Chu, J. F., Liu, X. W., and Wang, Y. M. (2016). Social network analysis based approach to group decision making problem with fuzzy preference relations. Journal of Intelligent and Fuzzy Systems $31,1271-1285$.

[8] De Cock, M. and Pinheiro Da Silva, P.(2006). A many-valued representation and propagation of trust and distrust. Lecture Notes in Computer Science 3849, 108-113.

[9] Dong, Y. C., Chen, X., and Herrera, F. (2015). Minimizing adjusted simple terms in the consensus reaching process with hesitant linguistic assessments in group decision making. Information Sciences 297, 95-117.

[10] Dong, Y. C., Ding, Z. G., Martínez, L. and Herrera, F. (2017). Managing consensus based on leadership in opinion dynamics. Information Sciences 397-398,187-205.

[11] Dong, Y. C., Ding, Z. G., Chiclana, F. and Herrera-Viedma, E. (2017). Dynamics of public opinions in an online and offline social network. IEEE Transactions on Big Data 99, 1-11. 
[12] Dong, Y. C., and Herrera-Viedma, E. (2015). Consistency-driven automatic methodology to set interval numerical scales of 2-tuple linguistic term sets and its use in the linguistic GDM with preference relation. IEEE Transactions on Cybernetics 45, 780-792.

[13] Dong, Y. C, Luo, N., and Liang, H. M. (2015). Consensus building in multiperson decision making with heterogeneous preference representation structures: A perspective based on prospect theory. Applied Soft Computing 35, 898-910.

[14] Dong, Y. C., Xu, Y. F., Li, H. Y. and Feng, B. (2010). The OWA-based consensus operator under linguistic representation models using position indexes. European Journal of Operational Research 203, 455-463.

[15] Dong, Y. C. and Xu, J. P.(2016). Consensus Building in Group Decision Making - Searching the Consensus Path with Minimum Adjustments. Springer-Verlag.

[16] Dong, Y. C, Zhang, H. J. and Herrera-Viedma, E. (2016). Integrating experts' weights generated dynamically into the consensus reaching process and its applications in managing non-cooperative behaviors. Decision Support Systems 84, 1-15.

[17] Gong, Z. W., Xu, X. X., Li, L. S., and Xu, C. (2015). Consensus modeling with nonlinear utility and cost constraints: A case study. Knowledge-Based Systems 88, 210-222.

[18] Gong, Z. W., Xu, X. X., Zhang. H. H., Ozturk, U. A. Herrera-Viedma, E., and Xu, C. (2015). The consensus models with interval preference opinions and their economic interpretation. Omega $55,81-90$.

[19] Gong, Z. W, Zhang, H. H., Forrest, J., Li, L. S., and Xu, X. X. (2015). Two consensus models based on the minimum cost and maximum return regarding either all individuals or one individual. Europen Journal of Operational Research 240, 183-192.

[20] González-Arteaga, T., de Andrés Calle, R. , and Chiclana, F. (2016) A new measure of consensus with fuzzy preference relations: The correlation consensus degree. Knowledge-Based Systems 10\%, $104-116$.

[21] Herrera-Viedma, E., Alonso, S., Chiclana, F., and Herrera, F. (2007). A consensus model for group decision making with incomplete fuzzy preference relations. IEEE Transactions on Fuzzy Systems 15(5), 863-877.

[22] Herrera-Viedma, E., Cabrerizo, F. J., Kacprzyk, J., and Pedrycz, W. (2014). A review of soft consensus models in a fuzzy environment. Information Fusion 17, 4-13. 
[23] Kacprzyk, J., Zadrozny, S., and Ras, Z. W. (2010) How to support consensus reaching using action rules: a novel approach. International Journal of Uncertainty, Fuzziness and KnowledgeBased Systems 36, 451-470.

[24] Kozierkiewicz-Hetmańska, A. (2017). The analysis of expert' opinions consensus quality. Information Fusion 34, 80-86.

[25] Lesser, A., Naamani-Dery, L., Kalech, M., and Elovici, Y. (2017). Group Decision Support for Leisure Activities Using Voting and Social Networks. Group Decision Negotiation 26, 473-494.

[26] Li, C. C., Dong, Y. C., Herrera, F., Herrera-Viedma, E., and Martínez, L. (2017). Personalized individual semantics in computing with words for supporting linguistic group decision making. An application on consensus reaching. Information Fusion 33, 29-40.

[27] Liang, Q., Liao, X. W., and Liu, J. P. (2017). A social ties-based approach for group decisionmaking problems with incomplete additive preference relations. Knowledge-Based Systems 119,6886.

[28] Liu, Y. and Fan, Z. P. (2016). A method for large group decision-making based on evaluation information provided by participators from multiple groups. Information Fusion 29, 132-141.

[29] Liu, Y. J., Liang, C. Y. Chiclana, F., and Wu, J. (2017). A trust induced recommendation mechanism for reaching consensus in group decision making. Knowledge-Based Systems 119, 221231.

[30] Mata, F., Martínez, L., and Herrera-Viedma, E. (2009). An adaptive consensus support system model for group decision-making problems in a multigranular fuzzy linguistic context. IEEE Transactions on Fuzzy Systems 17, 279-290.

[31] Meng, F. Y., An, Q. X., Tan, C. Q., and Chen, X. H. (2016). An approach for group decision making with interval fuzzy preference relations based on additive consistency and consensus analysis. IEEE Transactions on Systems, Man, and Cybernetics: Systems 99,1-14.

[32] Palomares, I., Estrella, F. J., Martínez, L., and Herrera, F. (2014). Consensus under a fuzzy context: Taxonomy, analysis framework AFRYCA and experimental case of study. Information Fusion 20, 252-271.

[33] Pérez, I. J., Cabrerizo, F. J., Alonso, S. and Herrera-Viedma, E. (2014). A New Consensus Model for Group Decision Making Problems with Non Homogeneous Experts. IEEE Transactions on Systems, Man, and Cybernetics: Systems 44 (4), 494-498. 
[34] Pérez, I. J.,Cabrerizo, F. J., and Herrera-Viedma, E. (2010). A Mobile Decision Support System for Dynamic Group Decision Making Problems. IEEE Transactions on Systems, Man and Cybernetics - Part A: Systems and Humans 40, 1244-1256.

[35] Pérez, L. G., Mata, F., and Chiclana, F. (2014). Social network decision making with linguistic trustworthiness based induced OWA operators. International Journal of Intelligent Systems 29, $1117-1137$.

[36] Pérez, L. G, Mata, F., Chiclana, F., Kou, G. and Herrera-Viedma, E. (2016). Modelling influence in group decision making. Soft Computing 20, 1653-1665.

[37] Quesada, F. J., Palomares, I., and Martínez, L. (2015). Managing experts behavior in large-scale consensus reaching processes with uninorm aggregation operators. Applied Soft Computing 35 (4), $873-887$.

[38] Roselló, L., Sánchez, M., Agell, N., Prats, F., and Mazaira, F. A. (2014). Using consensus and distances between generalized multi-attribute linguistic assessments for group decision-making. Information Fusion 17, 83-92.

[39] Sun, B. Z., and Ma, W. M. (2015). An approach to consensus measurement of linguistic preference relations in multi-attribute group decision making and application. Omega 51,83-92.

[40] Wasserman, S., and Faust, K. (1994). Social Network Analysis: Methods and Applications. Cambridge University Press.

[41] Wu, J., and Chiclana, F. (2014). Visual information recommendation simulation and attitudinal prioritisation method for group decision making with triangular fuzzy complementary preference relations. Information Sciences 279, 716-734.

[42] Wu, J. and Chiclana, F. (2014). Multiplicative consistency of intuitionistic reciprocal preference relations and its application to missing values estimation and consensus building. Knowledge-Based Systems 71, 187-200.

[43] Wu, J., and Chiclana, F. (2014). A social network analysis trust-consensus based approach to group decision-making problems with interval-valued fuzzy reciprocal preference relations. Knowledge-Based Systems 59, 97-107.

[44] Wu, J., Chiclana, F., Fujita, H., and Herrera-Viedma, E. (2017). A visual interaction consensus model for social network group decision making with trust propagation. Knowledge-Based Systems $39,39-50$.

[45] Wu, J., Chiclana, F., and Herrera-Viedma, E. (2015). Trust based consensus model for social network in an incomplete linguistic information context. Applied Soft Computing 35, 827-839. 
[46] Wu, J., Chiclana, F., and Liao, H. C. Isomorphic multiplicative transitivity for intuitionistic and interval-valued fuzzy preference relations and its application in deriving their priority vectors. IEEE Transactions on Fuzzy Systems doi:10.1109/TFUZZ.2016.2646749.

[47] Wu, J., Liu, Y. J., and Liang, C. Y. (2015). A consensus- and harmony-based feedback mechanism for multiple attribute group decision making with correlated intuitionistic fuzzy sets. International Transactions in Operational Research 22, 1033-1054.

[48] Wu, J., Xiong, R. Y., and Chiclana, F. (2016). Uninorm trust propagation and aggregation methods for group decision making in social network with four tuple information. Knowledge-Based Systems 96, 29-39.

[49] Xu, G. L., Wan, S. P., Wang, F., Dong, J. Y., Zeng, Y. F.(2016). Mathematical programming methods for consistency and consensus in group decision making with intuitionistic fuzzy preference relations. Knowledge-Based Systems 98, 30-43.

[50] Xu, J., Wan, S. P., and Dong, J. Y. (2016). Aggregating decision information into Atanassov's intuitionistic fuzzy numbers for heterogeneous multi-attribute group decision making. Applied Soft Computing 41,331-351.

[51] Xu, Z. S. (2005). Deviation measures of linguistic preference relations in group decision making. Omega 33, 249-254.

[52] Zhang, B. W., Dong, Y. C., and Xu, Y. F (2015). Multiple attribute consensus rules with minimum adjustments to support consensus reaching. Knowledge-Based Systems 67, 35-48.

[53] Zhang, G. Q., Dong, Y. C., and Xu, Y. F. (2014). Consistency and consensus measures for linguistic preference relations based on distribution assessments. Information Fusion 17, 46-55.

[54] Zhou, Y. Y., Chen, L. H., Zhou, L. G., Chen, H. Y., and Ge, J. Q. (2015). A group decision making approach for trapezoidal fuzzy preference relations with compatibility measure. Soft Computing 17, 1-13. 